\title{
Off-axis electron holography of magnetotactic bacteria: magnetic microstructure of strains MV-1 and MS-1
}

\author{
RAFAL E. DUNIN-BORKOWSKI ${ }^{1}$, MARTHA R. McCARTNEY ${ }^{2}$, MIHÁLY PÓSFAI $^{3}$, \\ RICHARD B. FRANKEL ${ }^{4}$, DENNIS A. BAZYLINSKI ${ }^{5}$ and PETER R. BUSECK ${ }^{6}$
}

\author{
${ }^{1}$ Department of Materials Science and Metallurgy, University of Cambridge, Pembroke Street, \\ Cambridge CB2 3QZ, UK \\ ${ }^{2}$ Center for Solid State Science, Arizona State University, Tempe, Arizona 85287-1704, USA \\ ${ }^{3}$ Department of Earth and Environmental Sciences, University of Veszprém, \\ Veszprém, POB 158, H-8201, Hungary \\ ${ }^{4}$ Department of Physics, California Polytechnic State University, San Luis Obispo, CA 93407, USA \\ ${ }^{5}$ Department of Microbiology, Immunology and Preventive Medicine, \\ Iowa State University, Ames, IA 50011, USA \\ ${ }^{6}$ Departments of Geological Sciences and Chemistry/ Biochemistry, Arizona State University, \\ Tempe, AZ 85287-1404, USA
}

\begin{abstract}
Off-axis electron holography in the transmission electron microscope is used to characterize the magnetic microstructure of magnetotactic bacteria. The practical details of the technique are illustrated through the examination of single cells of strains MV-1 and MS-1, which contain crystals of magnetite $\left(\mathrm{Fe}_{3} \mathrm{O}_{4}\right)$ that are $\sim 50 \mathrm{~nm}$ in size and are arranged in chains. Electron holography allows the magnetic domain structures within the nanocrystals to be visualized directly at close to the nanometer scale. The crystals are shown to be single magnetic domains. The magnetization directions of small crystals that would be superparamagnetic if they were isolated are found to be constrained by magnetic interactions with adjacent, larger crystals in the chains. Magnetization reversal processes are followed in situ, allowing a coercive field of between 30 and $45 \mathrm{mT}$ to be measured for the MV-1 cell. To within experimental error, the remanent magnetizations of the chains are found to be equal to the saturation magnetization of magnetite $(0.60 \mathrm{~T})$. A new approach is used to determine that the magnetic moments of the chains are 7 and $5 \times 10^{-16} \mathrm{Am}^{2}$ for the $1600-\mathrm{nm}$ long MV-1 and 1200-nm long MS-1 chains examined, respectively. The degree to which the observed magnetic domain structure is reproducible between successive measurements is also addressed.
\end{abstract}

Key-words: magnetotactic bacteria, off-axis electron holography, magnetite nanocrystals, biologically controlled mineralization.

\section{Introduction}

The transmission electron microscopy (TEM) technique of off-axis electron holography allows the amplitude and the phase shift of an electron wave that has passed through a sample (rather than its intensity) to be recorded (Tonomura, 1992a; Lichte, 1991). When studying magnetic materials, the most important of these parameters is the phase shift, as it can be used to obtain quantitative information about the magnetic field in the sample to a spatial resolution that can approach the nanometer scale. In this paper, we show how the magnetic microstructure of intracellular, membrane-bounded ferrimagnetic crystals, which are synthesized by magnetotactic bacteria and known as magnetoso- 
mes, can be characterized using off-axis electron holography. The magnetic crystals in magnetotactic bacteria are composed of magnetite $\left(\mathrm{Fe}_{3} \mathrm{O}_{4}\right)$ and/ or greigite $\left(\mathrm{Fe}_{3} \mathrm{~S}_{4}\right)$ and are usually arranged in one or more linear chains within each cell (Blakemore, 1975; Bazylinski \& Moskowitz, 1997). The magnetic moment that they impart to the cell results in the alignment and subsequent migration of the cell along the Earth's magnetic field lines (Frankel, 1984). This attribute is thought to increase the efficiency with which the cell finds an optimal position in a vertical chemical or redox gradient in an aquatic environment (Frankel et al., 1997).

Magnetotactic bacteria exercise a high degree of control over the morphologies of their constituent magnetosomes, which have a narrow size distribution, are specific to each cell type and usually have their magnetic easy axes aligned parallel to the chain axis (Bazylinski et al., 1994). The fact that the magnetic crystals are typically only $40-100 \mathrm{~nm}$ in size suggests that they should each contain a single magnetic domain (Moskowitz, 1995). However, magnetic force microscopy has insufficient resolution to characterize the magnetic microstructure of such crystals when they are still encapsulated within intact bacterial cells (Proksch et al., 1995). Differential phase contrast microscopy (Chapman et al., 1978; Daykin \& Petford-Long, 1995) is also poorly suited to the characterization of magnetic microstructure in sub-100-nm-sized nanocrystals because of large 'mean inner potential' contributions to the contrast from their edges (to be discussed further below). Here, we show that electron holography can be used to provide high spatial resolution, quantitative information about the magnetic microstructure of bacterial magnetosomes. The ability to obtain this information is crucial to understanding magnetic-field-sensing mechanisms in a wide range of organisms (Mann et al., 1988; Diebel et al., 2000), as well as providing magnetic biomarkers that may be used to establish the occurrence of ancient life (McKay et al., 1996; Thomas-Keprta et al., 2000). Magnetosomes also provide a model system for studying interacting single-domain magnetic crystals, which are of increasing interest to the electronics, catalysis and magnetic recording industries (Mann, 1993). Previous examples of the application of electron holography to magnetic materials have included the characterization of recording tapes (Tonomura, 1992b), Co particles (de Graef et al., 1999) and lithographically patterned nanostructures (Dunin-Borkowski et al., 1998a and 2000; Smith et al., 2000).
We begin by describing the basic experimental procedure required to obtain magnetic information from off-axis electron holograms of magnetic nanostructures. We then illustrate the specific precautions that are required when applying the technique to magnetotactic bacteria. The characterization of single cells of two strains of bacteria, designated MV-1 and MS-1, is described in detail. These strains contain magnetite crystals that have radically different morphologies. Qualitative results that reveal the magnetic microstructures of the magnetosome chains in the two cells are initially presented. Quantitative measurements of several of the cells' magnetic properties, including their magnetic moments, are then described. Finally, the results from the bacterial cells are compared with similar data obtained from lithographically patterned magnetic nanostructures.

Some of the results that are described in this paper have been presented in preliminary form elsewhere (Dunin-Borkowski et al., 1998b). However, the present paper provides a more detailed description of the experimental procedure required to apply electron holography specifically to magnetotactic bacteria. A novel approach for measuring the magnetic moment of a bacterial cell is also introduced.

\section{Experimental details}

Cultured cells of Magnetospirillum magnetotacticum strain MS-1 and the marine vibrioid strain MV-1 were prepared using methods that have been described by Frankel et al. (1997) and Bazylinski et al. (1988). Drops of water that had been enriched in bacteria were deposited directly onto 3-mm-diameter carbon or Formvar-coated Ni grids for TEM examination. Off-axis electron holography was performed at $200 \mathrm{kV}$ in a Philips CM200 field-emission-gun (FEG) TEM. This microscope has a rotatable electrostatic biprism (a $0.6 \mu \mathrm{m}$ quartz wire coated with gold) located in place of one of the conventional selected area apertures. It also has a Lorentz minilens, which is located in the bore of the objective lens pole-piece. The Lorentz minilens allows the examination of magnetic materials in close-to-field-free conditions (with the conventional microscope objective lens switched off) and has a line resolution of $1.2 \mathrm{~nm}$ at $200 \mathrm{kV}$.

All of the holograms presented below were recorded directly onto a $1024 \times 1024$ pixel charge-coupled-device (CCD) camera at a nominal microscope magnification of $30 \mathrm{k} \times$, corresponding to a field of 
view on the CCD camera of $630 \mathrm{~nm}$. A positive voltage of $120 \mathrm{~V}$ was applied to the biprism wire for holography. This biprism voltage was chosen because it provided an optimal holographic overlap width and fringe spacing (see below) of 640 and $3.9 \mathrm{~nm}$, respectively. Data analysis was performed on a Silicon Graphics workstation using library programs written in the Semper image processing language (Saxton et al., 1979). A Philips EM430 TEM equipped with a Gatan post-column imaging spectrometer was used to confirm the chemical compositions of the magnetite crystals under investigation. The chemical characterization of the samples is not described further in this paper.

\section{Background to off-axis electron holography}

The microscope geometry for off-axis electron holography is shown schematically in Fig. 1a. The sample is examined using defocused illumination from a FEG electron source, with the region of interest positioned so that it covers approximately half the field of view. A positive voltage of between 50 and $200 \mathrm{~V}$ is applied to an electrostatic biprism, which usually takes the form of either a Pt- or a $\mathrm{Au}$-coated quartz wire located in the selected area aperture plane of the microscope. This voltage causes the electron wave that has passed through the sample to overlap with a reference wave that has passed either through vacuum or through a thin region of the support film. The region of interest and the reference wave must be within $\sim 1 \mu \mathrm{m}$ of each other in order for the two waves to overlap coherently, and the orientation of the biprism wire may need to be optimized to satisfy this criterion. A low-magnification, bright-field image of the typical setup is shown in Fig. 1b. In this case, the biprism has been rotated to achieve overlap between a hole in the support film and a magnetosome chain from an MV-1 cell. The bright band of intensity running diagonally across the image is the overlap region of the two waves. The coarse fringes at the edges of the overlap region are Fresnel fringes from the edge of the biprism wire. At higher magnification, holographic interference fringes are visible within the overlap region, as shown for a hologram of a thin magnetic film in Fig. 1c. The spacing and the contrast of the fringes decrease with increasing biprism voltage.

The amplitude and the phase shift of the electron wave that leaves the sample are recorded in the intensity and the position of the holographic interfer- ence fringes, respectively. Experimentally, these parameters are obtained by extracting one 'sideband' from the Fourier transform of the hologram. This sideband is then inverse-Fourier-transformed, and the amplitude and phase of the resulting complex image wave are calculated, as shown in the lower half of Fig. 1c. Before further analysis the reconstructed phase image is 'unwrapped' to remove phase discontinuities, which result from the fact that it is initially calculated modulo $2 \pi$. A reference hologram is always obtained with the sample out of the field of view in order to remove artifacts associated with the imaging and recording process (de Ruijter \& Weiss, 1993). For magnetic materials, the use of a Lorentz lens allows samples to be examined in almost field-free conditions with the conventional microscope objective lens, which would result in a large vertical magnetic field at the position of the sample, switched off. When imaging using the Lorentz lens, the objective lens may also be excited slightly and the sample tilted in order to provide a component of the external field in the plane of the sample. In this way, magnetization processes can be followed in situ (Smith \& McCartney, 1999). The orientation of the sample may then need to be chosen to lie parallel to one of the tilt axes, and the applied magnetic field must be calibrated accurately (Fig. 1d). Further details about the practical requirements for electron holography, including information about recording and processing holograms, can be found elsewhere (Smith \& McCartney, 1999; Dunin-Borkowski et al., 1998c).

The recorded phase shift is sensitive to both the mean inner potential (MIP) of the sample and the in-plane component of the magnetic induction $\mathrm{B}$ integrated in the incident beam direction. Neglecting dynamical diffraction (i.e., assuming that no crystals are strongly diffracting), the phase shift can be expressed in the form

$$
\begin{aligned}
\phi(x)= & \left(\frac{2 \pi}{\lambda}\right)\left(\frac{E+E_{0}}{E\left(E+2 E_{0}\right)}\right) \int V(x, z) d z \\
& -\left(\frac{e}{h}\right) \iint B_{\perp}(x, z) d x d z
\end{aligned}
$$

where $\mathrm{z}$ is the incident electron beam direction, $\mathrm{x}$ is a direction in the plane of the sample, $B_{\downarrow}$ is the component of the magnetic induction perpendicular to both $\mathrm{x}$ and $\mathrm{z}, \mathrm{V}$ is the MIP in the sample, $\lambda$ is the wavelength and $\mathrm{E}$ and $\mathrm{E}_{0}$ are, respectively, the kinetic and rest mass energies of the incident electron (Reimer, 1991). The MIP, which depends on the local composition and density in the sample, can be expressed in the form 

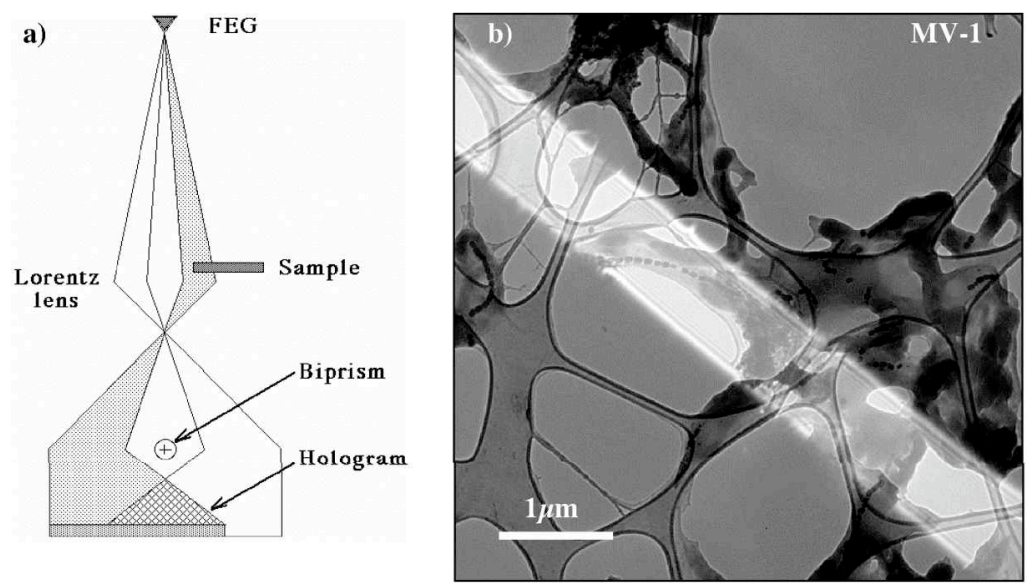

c)

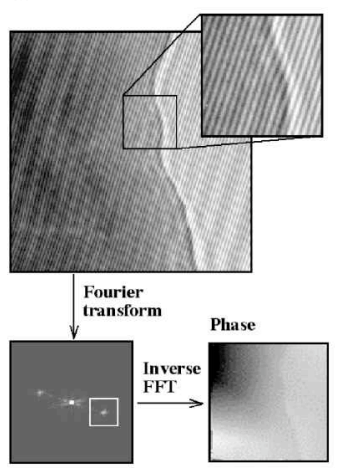

d)

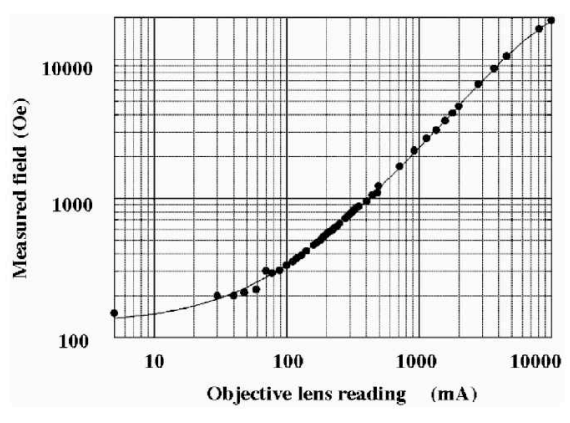

Fig. 1. a) Schematic illustration of setup used to generate off-axis electron holograms in the TEM. The sample occupies approximately half the field of view and is coherently illuminated by a field-emission electron gun (FEG). A positively charged electrostatic biprism (a thin gold-coated quartz fiber, $<0.7 \mu \mathrm{m}$ in diameter) is used to overlap the object and (vacu$\mathrm{um}$ ) reference waves. The resulting holographic interference pattern is recorded digitally. A Lorentz lens allows imaging of magnetic materials in close-to-field-free conditions. b) Low-magnification bright-field image of a single cell of magnetotactic bacterium strain MV-1 (located next to a hole in the carbon support film), which was obtained using the Lorentz lens and shows the effect on the image of applying $120 \mathrm{~V}$ to the biprism wire. c) Representative off-axis electron hologram from a thin crystal. The coarse fringes are Fresnel fringes from the edge of the biprism wire and the fine fringes (shown in the enlargement) are holographic interference fringes that carry information about the amplitude and phase of the electron wave at the exit surface of the sample. The Fourier transform of the electron hologram and the reconstructed phase image obtained after inverse Fourier transforming one 'sideband' from the Fourier transform are also shown. d) Hall probe calibration of the magnetic field in the specimen plane of the Philips CM200 FEGTEM, plotted as a function of objective lens current. The magnetic field is parallel to the incident beam direction and is insensitive to small changes in specimen height.

$\mathrm{V}=\left(\frac{\mathrm{h}^{2}}{2 \pi \mathrm{me} \Omega}\right) \sum_{\substack{\text { unit } \\ \text { cell }}} \mathrm{f}_{\mathrm{el}}(0)$

where $f_{e l}(0)$ is the electron scattering factor at zero scattering angle for each atom in the unit cell $\left(\mathrm{f}_{\mathrm{el}}(0)\right.$ is approximately proportional to atomic number) and $\Omega$ is the unit cell volume. If neither $V$ nor $B_{\perp}$ vary in the incident beam direction, Equation (1) can be simplified to:

$$
\begin{aligned}
\phi(x)= & \left(\frac{2 \pi}{\lambda}\right)\left(\frac{\mathrm{E}+\mathrm{E}_{0}}{\mathrm{E}\left(\mathrm{E}+2 \mathrm{E}_{0}\right)}\right) \mathrm{V}(\mathrm{x}) \mathrm{t}(\mathrm{x}) \\
& -\left(\frac{\mathrm{e}}{\hbar}\right) \int \mathrm{B}_{\perp}(\mathrm{x}) \mathrm{t}(\mathrm{x}) \mathrm{dx}
\end{aligned}
$$

where $t$ is the sample thickness. Differentiation with respect to $\mathrm{x}$ then leads to 


$$
\begin{aligned}
\frac{d \phi(x)}{d x}= & \left(\frac{2 \pi}{\lambda}\right)\left(\frac{E+E_{0}}{E\left(E+2 E_{0}\right)}\right) \frac{d}{d x}\{V(x) t(x)\} \\
& -\left(\frac{e}{\hbar}\right) B_{\perp}(x) t(x)
\end{aligned}
$$

For a sample of uniform thickness and composition, the first term in Equation (4) disappears and the phase gradient is directly proportional to the in-plane induction. A direct picture of the magnetic field lines in the sample can then be provided by adding contours to the measured phase image.

Experimentally, the spatial resolution of a recorded phase image is determined primarily by the choice of magnification and biprism voltage used. However, it may also be affected by the presence of noise, which results from the weak intensity of the magnetic signal being measured.

\section{The application of electron holography to magnetotactic bacteria}

In nanostructured magnetic elements or small magnetic crystals, the MIP term in Equations (3) and (4) invariably dominates both the phase and the phase gradient, complicating attempts to quantify the magnetization. This effect is usually most apparent at the edges of small crystals in a phase gradient image. (It is also particularly detrimental for differential phase contrast microscopy, which records the phase gradient directly rather than the phase). Additional steps are therefore required to separate the magnetic contribution to an electron holographic phase image $\phi_{\text {mag }}$ from the MIP contribution $\phi_{\text {mip }}$ (Dunin-Borkowski et al., 1998a). This separation can be achieved in several different ways: for the magnetotactic bacteria studied here, we have chosen to record two holograms with the chain of crystals magnetized in opposite directions. On the assumption that the magnetic field in the chain has reversed exactly, $\phi_{\text {mip }}$ and $\phi_{\text {mag }}$ can be obtained simply by taking half the difference and half the sum of the phases of the two holograms, respectively (Dunin-Borkowski et al., 1998a). This procedure also ensures that dynamical contributions from any strongly diffracting crystals are removed from $\phi_{\text {mip }}$ (Dunin-Borkowski et al., 1998d).

It should be noted that a sample that contains magnetic nanocrystals does not have uniform thickness or composition everywhere, and in addition B will vary in the incident electron beam direction as a result of the presence of stray magnetic fields from each crystal. The phase gradient will therefore not be directly proportional to the in-plane induction. However, the addition of contours to the measured magnetic contribution to the phase $\phi_{\text {mag }}$ can still be used to provide a useful, albeit qualitative, picture of the magnitude and direction of the local magnetic field in the sample, as will be shown below.

Fig. 2a shows a bright-field image of the chain of MV-1 crystals examined. This chain is $1600 \mathrm{~nm}$ long, has 15 crystals that are each $\sim 60 \times \sim 35 \mathrm{~nm}$ in size, and is adjacent to a hole in the $\mathrm{C}$ support film. Previous studies have shown that the crystals in MV-1 have shapes that are formed from a combination of [110] (dodecahedron) and [111] (octahedron) faces and are elongated along $<111>$ (Meldrum et al., 1993). Fig. 2b shows a corresponding bright-field image of an MS-1 cell. The 1200-nm long chain forms the 'backbone' to the helical cell. Excluding the smallest crystals at its ends, it contains 22 crystals that have an average diameter and an average separation of $\sim 45$ and $\sim 9.5 \mathrm{~nm}$, respectively. Each crystal in MS- 1 has a shape that is formed from a combination of [111] (octahedron) and [100] (cube) faces. As for MV-1, the <111> axes of elongation of the crystals are usually parallel to the chain axis (Mann et al., 1984). The MS-1 cell is located on a continuou support film, and so the reference wave for each hologram comes from a region of the film rather than from vacuum.

At a magnification of $30 \mathrm{k} \times$, four holograms are required to span the length of each chain. Enlargements of individual holograms are shown in Fig. 2c and $2 \mathrm{~d}$ for the MV-1 and MS-1 chains, respectively. In each hologram, the phase changes of interest are visible in the form of bending of the holographic interference fringes as they pass through each crystal. This bending can be seen most clearly by looking along the fringes at a shallow angle to the plane of the paper.

In order to reverse the direction of magnetization in each chain in situ, the samples were tilted in the microscope by either $+30^{\circ}$ or $-30^{\circ}$. The objective lens was then turned on to provide a large (> $1 \mathrm{~T}$ ) component of the applied field parallel to the axis of each chain, as shown schematically in Fig. 2e. The objective lens was then turned off and the samples were tilted back to $0^{\circ}$ for hologram acquisition in the small residual vertical field of $\sim 13 \mathrm{mT}$ (see Fig. 1d). Examples of unwrapped phase images of a section of the MV-1 cell, between which the magnetization direction of the chain has been reversed, are shown in Fig. $2 \mathrm{f}$ and $2 \mathrm{~g}$. The two images are almost identical because they are both dominated by the MIP contribution to the phase. The change in magnetization direction 

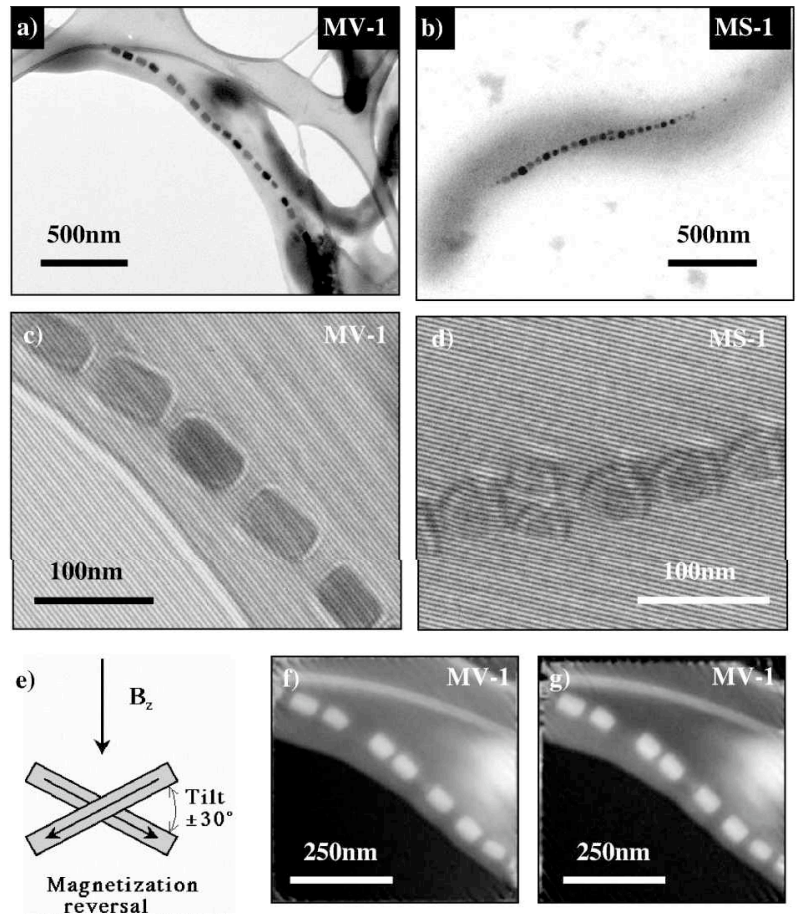

Fig. 2. TEM bright-field images of single cells of a) vibrioid strain MV-1 (containing a 1600$\mathrm{nm}$ chain of $15 \sim 60 \times \sim 35 \mathrm{~nm}$ magnetite crystals that are elongated along $<111>$ ) and b) Magnetospirillum magnetotacticum strain MS-1 (containing a $1200-\mathrm{nm}$ chain of 2245 -nm cuboctahedral magnetite crystals). c) and d) Small regions extracted from off-axis electron holograms acquired from the chains shown in a) and b), respectively. The holograms were obtained at a biprism voltage of $120 \mathrm{~V}$ using the Lorentz lens after tilting each sample in order to magnetize the chain along its axis. e) Schematic diagram illustrating the use of sample tilt to provide a component of the applied field in the plane of the sample. f) and g) Phase images reconstructed from holograms of the same region of the MV-1 chain shown in a), between which the magnetization direction of the chain was reversed. results only in a slight change in background intensity across the chain; as a result, the top right of Fig. $2 g$ is very slightly darker than the equivalent part of Fig. 2f. Pairs of images such as those shown in Fig. $2 \mathrm{f}$ and $2 \mathrm{~g}$ were used to obtain all of the magnetic information presented below.

\section{Qualitative observations}

\section{a) Magnetic domain structure}

The MIP and magnetic contributions to the measured phase shift are shown in Fig. 3a and 3b, respectively, for the entire length of the MV-1 chain. The figures were calculated from 16 separate images (4 pairs of holograms of the sample and 4 pairs of reference holograms). By definition, the spatial resolution of Fig. 3a and $3 \mathrm{~b}$ can be no better than twice the holographic fringe spacing used, or $7.8 \mathrm{~nm}$. Both the crystals and the support film are delineated clearly in Fig. 3a, which could in principle be used to determine the morphology of each crystal. In comparison, the features in Fig. 3b vary much more slowly. Figure $3 \mathrm{c}$ shows contours of spacing 0.064 radians that have been formed from Fig. 3b. They have also been overlaid onto Fig. 3a so that the positions of the crystals can be identified clearly. (The same crystal is marked with an arrow in Fig. 3a and 3c). The contours provide a semi-quantitative map of the strength of the local magnetic field in the sample. Strictly, their spacing is (inversely) proportional to the component of the magnetic induction in the plane of the sample integrated in the incident beam direction. As predicted, all of the crystals in the MV-1 chain are single magnetic domains that are magnetized approximately parallel to the chain axis. The contours are most closely-spaced within the crystals, where the field is strongest, and increase in separation both at the ends of the chain and in the larger gaps between individual magnetosomes. The observation that the contours 'fan out' is suggestive of the presence of a 'flower' magnetization state in the crystal at the end of the chain.

Equivalent images for the MS-1 chain are shown in Fig. 4a-c. Fig. 4a shows that the MS-1 chain is asymmetrical, with larger crystals at its left end. It is interesting to note that the size of the crystals decreases more gradually at the right end of the chain and more discontinuously at its left end. The chain also contains a double-crystal defect, which is arrowed in Fig. 4a and 4c. As for the MV-1 chain, the morphologies and the orientations of the individual crystals can be identified in Fig. 4a, while the 
Fig. 3. a) Mean inner potential and b) magnetic contributions to the reconstructed phase of the single cell of MV-1 shown in Fig. 2a. Each image is a montage that was created from either the sum or the difference of four pairs of phase images such as those shown in Fig. $2 \mathrm{f}$ and $2 \mathrm{~g}$ (see text for details). c) Contours of spacing 0.064 radians, formed from the magnetic contribution to the holographic phase, overlaid onto the mean potential contribution to the phase (to allow the positions of the crystals to be correlated with the magnetic contours). The contours lie parallel to lines of constant magnetic induction integrated in the incident beam direction. They are closest together within the crystals, spread out both at the end of the chain and at gaps between individual crystals, and are most closely spaced where the thickness of the crystals is greatest. The intensity of the background differs between the two sides of the chain because of variations in the projected thickness of the cell.
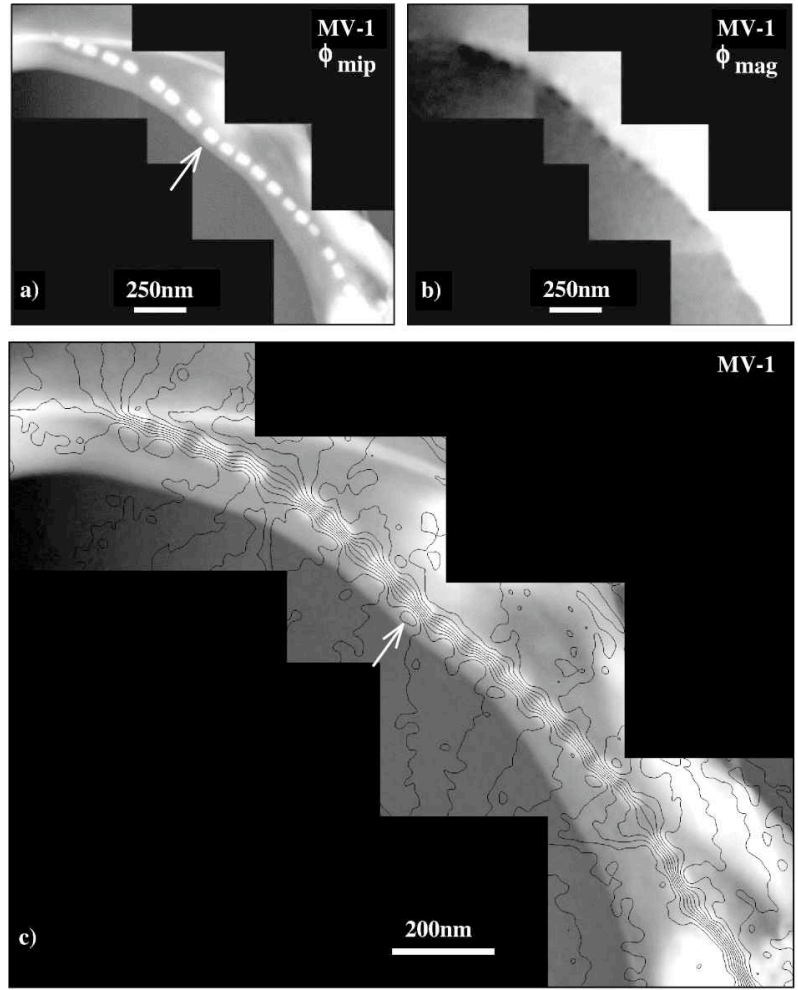

magnetic contribution to the phase in Fig. 4b again varies slowly across the image. The local variation in $\phi_{\mathrm{mag}}$ is more rapid in the left half of the chain, which contains the larger crystals. As a result, the contour map in Fig. 4c shows a larger number of closely-spaced contours at the left end of the chain. The contours can also be seen to bend within some of the crystals in order to lower their magnetostatic energy, whereas in others their direction differs slightly from that of the chain axis. These points will be discussed in more detail below.

Schematic diagrams that show the forms of $\phi_{\text {mip }}$ and $\phi_{\text {mag }}$ as surface plots are drawn in Fig. 5a and $5 \mathrm{~b}$, respectively, for a helical MS-1 cell that is magnetized parallel to the positive y-axis. (Fig. 5c and $5 \mathrm{~d}$ are described in Section 6). Fig. 5a and $5 \mathrm{~b}$ are intended to illustrate the rapid variation in $\phi_{\text {mip }}$ due to the crystals, and the fact that a slight background is present due to the organic material that surrounds them. Fig. 5b illustrates the fact that, for crystals that are all single magnetic domains oriented along a chain of finite length, the gradient of $\phi_{\text {mag }}$ varies most rapidly at the crystals and then dies away to zero in all directions. Schematic line profiles taken along the lines $\mathrm{X}-\mathrm{X}^{\prime}$ and $\mathrm{Y}-\mathrm{Y}^{\prime}$ ' in Fig. 5a and $5 \mathrm{~b}$ are shown in Fig. 6a and 6b, respectively. Similar profiles have been presented for individual crystals by de Graef et al. (1999).

The identification of single magnetic domains in the two cells in Fig. $3 \mathrm{c}$ and $4 \mathrm{c}$ is consistent with a qualitative prediction of a single- to multi-domain transition when the size of the crystals is comparable to the domain wall width of $\sim 100 \mathrm{~nm}$ in bulk magnetite (Dunlop \& Özdemir, 1997). The precise particle size at which this transition occurs, as well as the external factors that may affect it, has been uncertain.

Numerical micro-magnetic modeling predicts that the transition should occur at a size of $\sim 70 \mathrm{~nm}$ for cubes (Fabian et al., 1996), although this value is predicted to increase with axial ratio (Fabian et al., 1996; Bazylinski \& Moskowitz, 1997). The situation is complicated by the fact that metastable single domains may exist in the multi-domain regime (Moskowitz, 1995), particularly in the presence of interactions between closely spaced particles.

\section{Defects}

Electron holography allows the effect of defects on the magnetic microstructure of magnetosome 

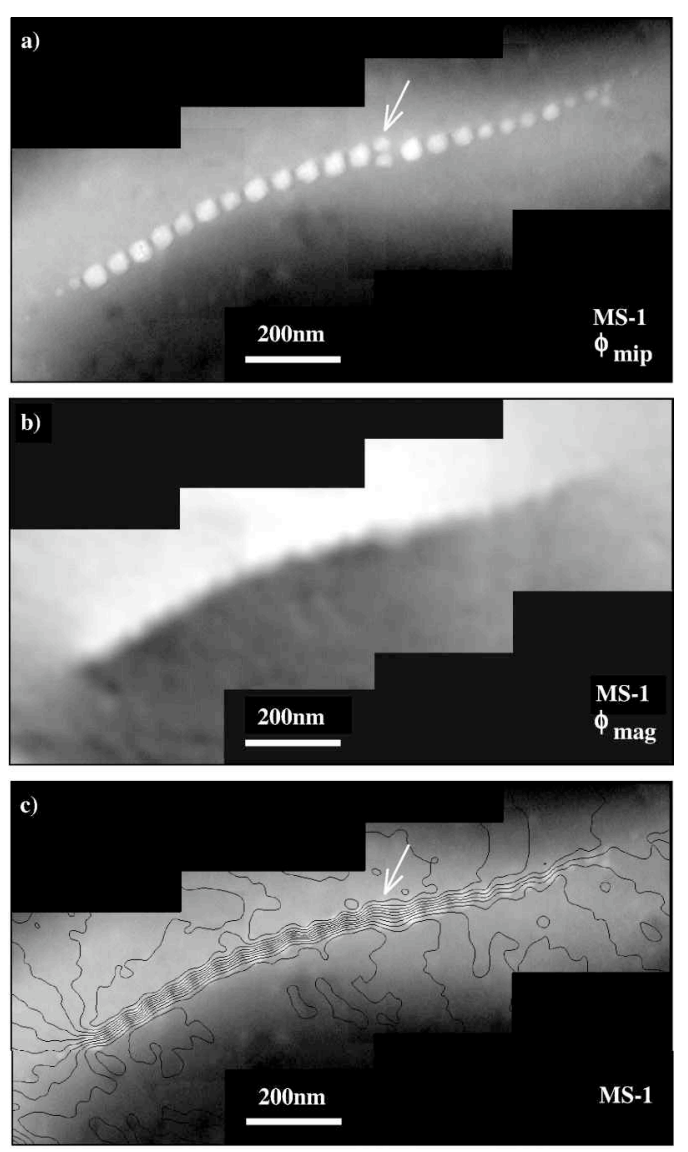

Fig. 4. As for Fig. 3 but for the single cell of MS-1 shown in Fig. 2b.

chains to be studied directly. Defects may include variations in the orientations or positions of the crystals (see below) or the presence of crystallographic twins or variations in chemistry within individual crystals. The defect in the MS-1 chain shown in Fig. 4 results from the replacement of a single large magnetite crystal by two smaller crystals. The contour map in Fig. 4c confirms the fact that the smaller crystals are magnetized (the local contour separation would be much wider if they were not). Although the presence of the crystals results in the slightly poorer confinement of the field lines, it does not significantly affect the magnetic microstructure or the total magnetic dipole moment of the cell. In the MV-1 cell shown in Fig. 3, the large gap between the fourth and fifth crystals from the left end of the chain also affects the confinement of the field lines very little, and the consequent decrease in the magnetic moment of the cell is negligible.

\section{Superparamagnetic crystals}

The crystals at the right end of the MS- 1 chain in Fig. 4 are small enough to be superparamagnetic if they were isolated. At room temperature, this transition should occur when the crystals are below approximately 20 to $35 \mathrm{~nm}$ in size (Dunlop \& Özdemir, 1997; Bazylinski \& Moskowitz, 1997). As a result, their magnetization direction should fluctuate rapidly and their measured magnetization should be zero. However, the contours in Fig. $4 \mathrm{c}$ are closely spaced through these crystals, indicating that they are still magnetized approximately parallel to the chain axis. Therefore, either the expected thermal fluctuations in magnetization are overcome by interactions with the field of the larger crystals in the chain, or the measurement time (the acquisition time for a hologram) is shorter than the time over which the magnetization direction in the crystals fluctuates. The fact that they are magnetic also indicates that non-magnetic precursors, which have been identified in magnetotactic bacteria with greigite $\left(\mathrm{Fe}_{3} \mathrm{~S}_{4}\right)$ crystals in their magnetosomes (Pósfai et al., 1998), have either not formed during magnetite synthesis in this MS-1 cell or have already transformed to a magnetic form on the sample grid. The presence or absence of non-magnetic precursors is clearly important if bacterial magnetosomes are to be used as biomarkers.

\section{Anisotropy}

In the MV-1 chain, the direction of the contours in the fourth magnetosome from the left is misaligned with respect to its neighbor (Fig. 3c), suggesting that the magnetization direction in this crystal is dominated by its shape rather than by the direction of the chain axis. In the MS- 1 chain shown in Fig. $7 \mathrm{a}$ and $7 \mathrm{~b}$, the morphologies of the crystals (Fig. 7a) indicate that they are aligned with their $<111>$ magnetic easy axes along the chain axis, whereas the magnetization direction within two crystals that are offset slightly from the others differs from both their $<111\rangle$ direction and the chain axis (Fig. 7b). These observations suggest that the difference between the behavior for MV-1 and MS-1 is a direct result of the weak magnetocrystalline anisotropy of magnetite, with shape anisotropy playing a dominant role in MV-1. (Magnetite has a low value for the first anisotropy constant, $\mathrm{K}_{1}$, of $1.35 \times 10^{4} \mathrm{Jm}^{-3}$, about $30 \%$ of that of $\mathrm{Fe}$ ). However, this conclusion is inconsistent with Fig. 7c and 7d, in which the magnetization direction of the left hand crystal (which is the last crystal in the chain) 
Fig. 5. Schematic diagrams illustrating possible contributions to the measured electron holographic phase of a magnetosome chain within a bacterial cell, as well as certain parameters that may be used to calculate the cell's magnetic properties. A three-dimensional representation of a magnetosome chain within a single helical bacterial cell is shown at the top of the figure, with the incident electron beam oriented along the negative $\mathrm{z}$-direction. The direction of magnetization of the chain (along the $y$-axis) is shown by the arrow. a) Surface plot showing the mean inner potential contribution to the phase. The darker spikes correspond to the magnetic nanocrystals. b) Surface plot showing the corresponding magnetic contribution to the phase; c) and d) Derivatives of the surface plot shown in b), which are used to determine the magnetic moment of the cell in two different directions (see text for details).

Fig. 6. Line profiles illustrating the forms of the surface plots shown in Fig. 5a-5d along the lines $X-X^{\prime}$ and Y-Y'. The parameter $\Delta \phi_{\text {mag }}$, which may be used in calculations of the cell's magnetic moment, is defined in b).

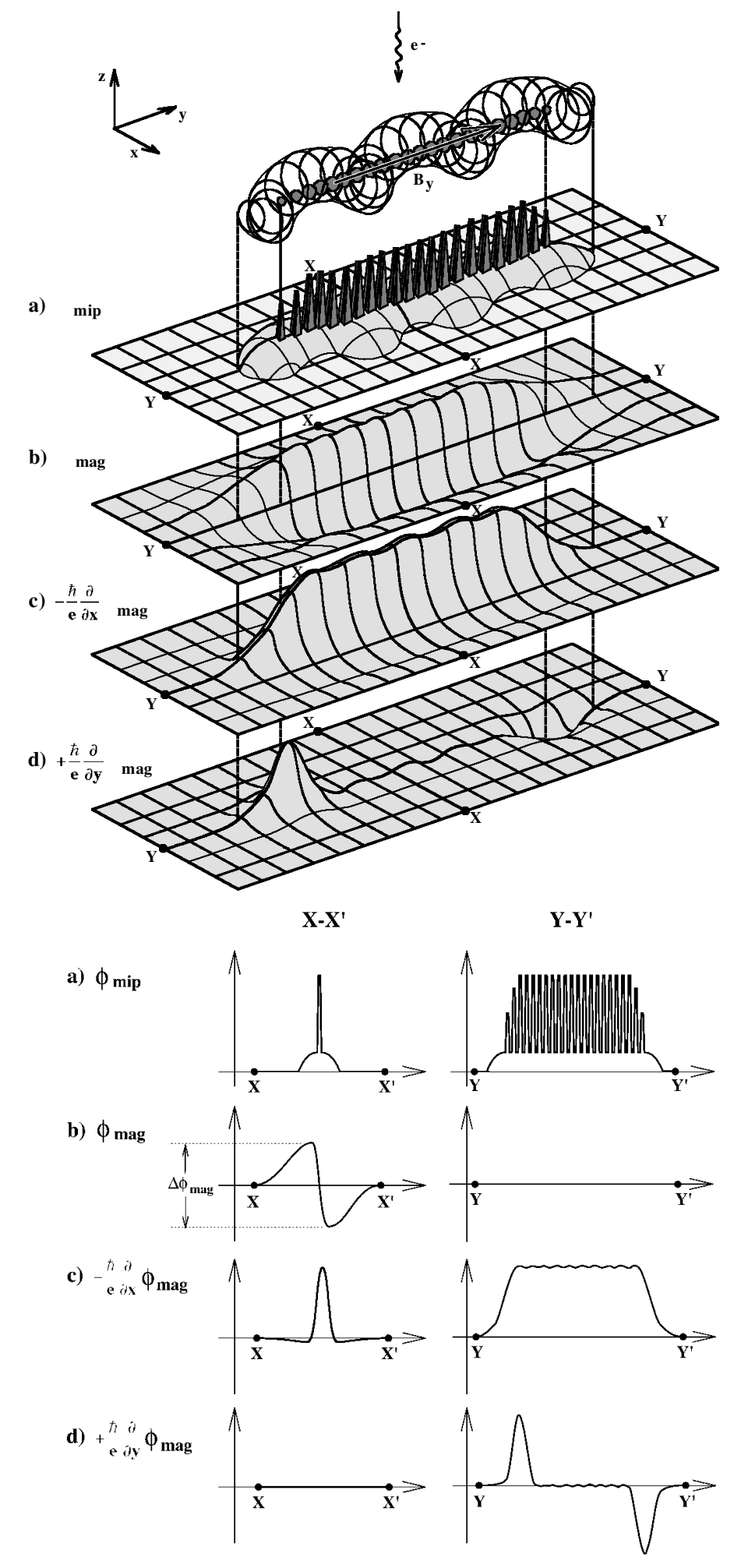



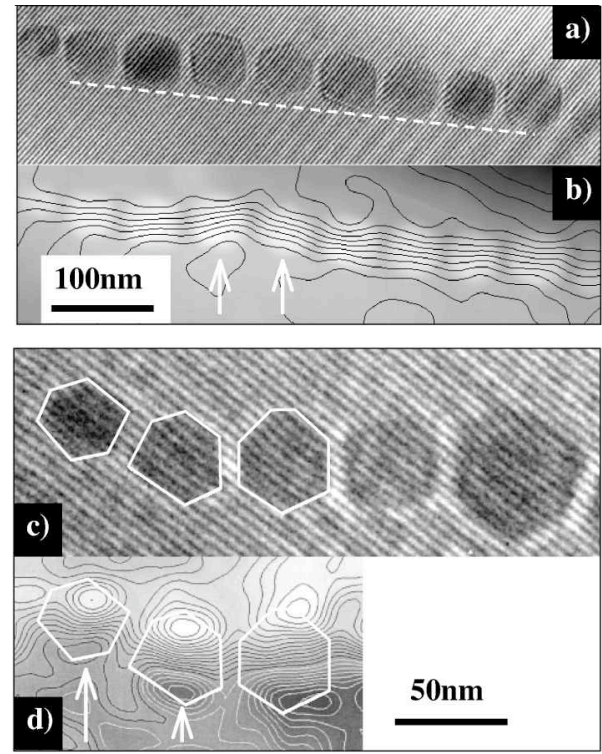

Fig. 7. a) Electron hologram of part of a magnetosome chain within a cell of strain MS-1. A line is drawn as a visual aid to show that the two crystals arrowed are offset slightly from the chain axis. b) Contours formed from the magnetic contribution to the holographic phase overlaid onto the mean inner potential inner contribution to phase. c) and d) As for a) and b) but for a different magnetosome chain.

is misaligned from the other crystals in the chain. Both further experimental studies and micromagnetic simulations are required to understand the interplay between magnetocrystalline and shape anisotropy in magnetite crystals of this size.

\section{Reproducibility}

The approach that we have used to determine the magnetic contribution to the measured holographic phase $\phi_{\text {mag }}$ has required the analysis of two sets of images between which the magnetization direction of the chain is reversed. This approach relies on the assumption that the local magnetization direction in the magnetosome chain reverses without changing in detail. Ideally, several measurements should therefore be made to ensure that the observations are consistent between successive measurements. The results of such an experiment are shown in Fig. 8 for the MS-1 chain shown in Fig. 7c and 7d. Fig. $8 \mathrm{a}$ and $8 \mathrm{~b}$ were calculated using datasets from different magnetization reversal experiments. Although the precise position s of the contours are different between the two figures, it is encouraging to notice that the details of the magnetic domain struc-

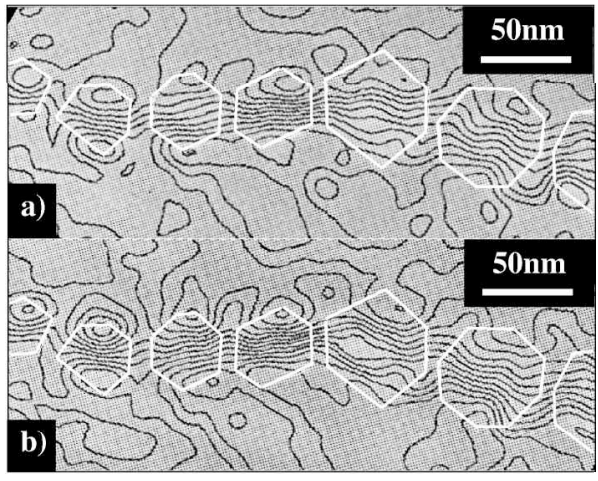

Fig. 8. Contours formed from the magnetic contribution to the holographic phase for the MS-1 magnetosome chain shown in Fig. 7c and d. a) and b) show the results of two separate magnetization reversal experiments.

ture are reproduced accurately between the two experiments, both within the crystals and in the surrounding magnetic fringing fields.

\section{Quantitative measurements}

Although the contours shown in Fig. $3 \mathrm{c}$ and $4 \mathrm{c}$ provide a wealth of information, one of the primary strengths of electron holography is its ability to provide quantitative information about magnetic properties.

\section{Magnetic moment}

One of the most straightforward parameters that can be obtained from the magnetic contribution to the phase is the magnetic moment of a bacterial cell. Two approaches for measuring this parameter are now outlined.

By definition, the magnetic moment of a bacterial cell (or any other magnetic nanostructure) along the $\mathrm{x}$ and $\mathrm{y}$ directions is given by the relations

$m_{x}=\int_{-\infty}^{+\infty} \int_{-\infty}^{+\infty} \int_{-\infty}^{+\infty} B_{x}(x, y, z) d x d y d z$

and

$\mathrm{m}_{\mathrm{y}}=\int_{-\infty}^{+\infty} \int_{-\infty}^{+\infty} \int_{-\infty}^{+\infty} \mathrm{B}_{\mathrm{y}}(\mathrm{x}, \mathrm{y}, \mathrm{z}) \mathrm{dxdydz}$,

respectively. However, according to Equation (1)

$\phi_{\operatorname{mag}}(x, y)=-\left(\frac{\mathrm{e}}{\hbar}\right)_{z=-\infty}^{\mathrm{z}=+\infty} \int_{\mathrm{x}= \pm \infty}^{\mathrm{x}} \mathrm{B}_{\mathrm{y}}(\mathrm{x}, \mathrm{y}, \mathrm{z}) \mathrm{dxd} \mathrm{z}$ 


$$
\equiv+\left(\frac{\mathrm{e}}{\hbar}\right)_{\mathrm{z}=-\infty}^{\mathrm{z}=+\infty} \int_{\mathrm{y}= \pm \infty}^{\mathrm{y}} \mathrm{B}_{\mathrm{x}}(\mathrm{x}, \mathrm{y}, \mathrm{z}) \mathrm{dyd} \mathrm{z} .
$$

It follows from Equations (7) and (8) that

$$
\begin{aligned}
& \frac{\partial}{\partial x} \phi_{\operatorname{mag}}(x, y)=-\left(\frac{e}{\hbar}\right)_{z=-\infty}^{z=+\infty} B_{y}(x, y, z) d z \\
& \frac{\partial}{\partial y} \phi_{\operatorname{mag}}(x, y)=+\left(\frac{e}{\hbar}\right)_{z=-\infty}^{z=+\infty} B_{x}(x, y, z) d z .
\end{aligned}
$$

Combining Equations (5), (6), (9) and (10), the equations for the magnetic moment can be expressed in the form:

$\mathrm{m}_{\mathrm{x}}=+\left(\frac{\hbar}{\mathrm{e}}\right)_{\mathrm{y}=-\infty}^{\mathrm{y}=+\infty} \int_{\mathrm{x}=-\infty}^{\mathrm{x}=+\infty} \frac{\partial}{\partial \mathrm{y}} \phi_{\text {mag }}(\mathrm{x}, \mathrm{y}) \mathrm{dxdy}$

and

$\mathrm{m}_{\mathrm{y}}=-\left(\frac{\hbar}{\mathrm{e}}\right)_{\mathrm{y}=-\infty}^{\mathrm{y}=+\infty} \int_{\mathrm{x}=-\infty}^{\mathrm{x}=+\infty} \frac{\partial}{\partial \mathrm{x}} \phi_{\operatorname{mag}}(\mathrm{x}, \mathrm{y}) \mathrm{dxdy}$

Equations (11) and (12) show that the magnetic moment of a bacterial cell in a given direction can be obtained by measuring the area under the first differential of the magnetic contribution to the phase evaluated in the perpendicular direction. Schematic diagrams illustrating Equations (11) and (12) in the form of surface plots are shown in Fig. 5c and 5d, respectively. The magnetic moment of the cell along $y$ is obtained by measuring the area under Fig. 5c, while the moment along $\mathrm{x}$ is obtained from the area under Fig. $5 \mathrm{~d}$ and yields the expected value of zero. Corresponding line profiles along $X-X^{\prime}$ and Y-Y' are shown in Fig. 6c and 6d. The application of Equation (12) to the MV-1 and MS-1 cells examined above gives magnetic moments (along the average directions of the chain axes) of $(7.5 \pm 0.3) \times 10^{-16} \mathrm{Am}^{2} \quad\left(7.5 \times 10^{-13} \mathrm{emu}\right)$ and $(5.2 \pm 0.2) \times 10^{-16} \mathrm{Am}^{2}\left(5.2 \times 10^{-13} \mathrm{emu}\right)$, respectively.

An alternative, simpler approach for measuring the moment of a cell results from the fact that, to a good approximation, Equation (12) can be rewritten in the form

$\mathrm{m}_{\mathrm{y}} \approx+\left(\frac{\hbar}{\mathrm{e}}\right)_{\text {chain }} \Delta \phi_{\text {mag }}(\mathrm{x}, \mathrm{y}) \mathrm{dl}$

where $\Delta \phi_{\text {mag }}$ is the local change in the magnetic contribution to the phase across the chain (defined in Fig. 6b) and 1 is a direction along the chain axis. The moment can therefore be calculated by multiplying the average value of $\Delta \phi_{\mathrm{mag}}$ along the chain by its length. This approach gives moments for the
MV -1 and MS- 1 cells of $(7 \pm 1) \times 10^{-16} \mathrm{Am}^{2}\left(7 \times 10^{-13}\right.$ $\mathrm{emu})$ and $(5 \pm 1) \times 10^{-16} \mathrm{Am}^{2}\left(5 \times 10^{-13} \mathrm{emu}\right)$, respectively, in good agreement with the more accurate approach described above. Significantly, both approaches give moments per unit length that are the same for both chains to within experimental error, suggesting that the different crystal morphologies in MV-1 and MS-1 do not arise for magnetic reasons. However, this inference may not apply to magnetosome chains that contain considerably larger crystals, such as those reported by Farina et al. (1994), which would lie in the multi-domain régime if they were isolated.

\section{Coercive field}

The magnetization reversal process in a magnetosome chain may be followed in situ by obtaining holograms at successive values of the applied magnetic field. For example, measurements may be made at successive sample tilts with the objective lens excited to provide a known component of the applied field in the plane of the sample. We used this approach to measure the coercive field of the MV-1 cell (the in-plane component of the applied field at which the magnetization direction of the chain reversed). By monitoring the difference in the measured phase shift across the chain as a function of applied field, the coercive field was measured to be between 30 and $45 \mathrm{mT}$ ( 300 and $450 \mathrm{Oe}$ ). This value is consistent with a bulk coercive field measurement of $30 \mathrm{mT}$ made on freeze-dried cells of MV-1 (Moskowitz et al., 1989), as well as with the work of Penninga et al. (1995) who measured an average coercive field of $30 \mathrm{mT}$ for the MS-1 strain. The sensitivity of the coercive field to the size, separation, morphology and the number of crystals in a chain (Jacobs $\&$ Bean, 1955), as well as to magnetocrystalline anisotropy, highlights the importance of studying individual magnetosome chains rather than obtaining statistical measurements from large numbers of bacteria. A comparison of our measurement with a chain-of-spheres calculation for magnetite (Kuo, 1988) suggests that magnetization reversal proceeds by a symmetric fanning mechanism rather than by the parallel rotation of the magnetic moments of the crystals. Future studies using electron holography will allow this conclusion to be verified directly.

\section{Magnetization}

Measurements of either the magnetic moment of a magnetosome chain parallel to its axis (see Fig. 6b) or the parameter $\Delta \phi_{\text {mag }}$ can be used to determine the 

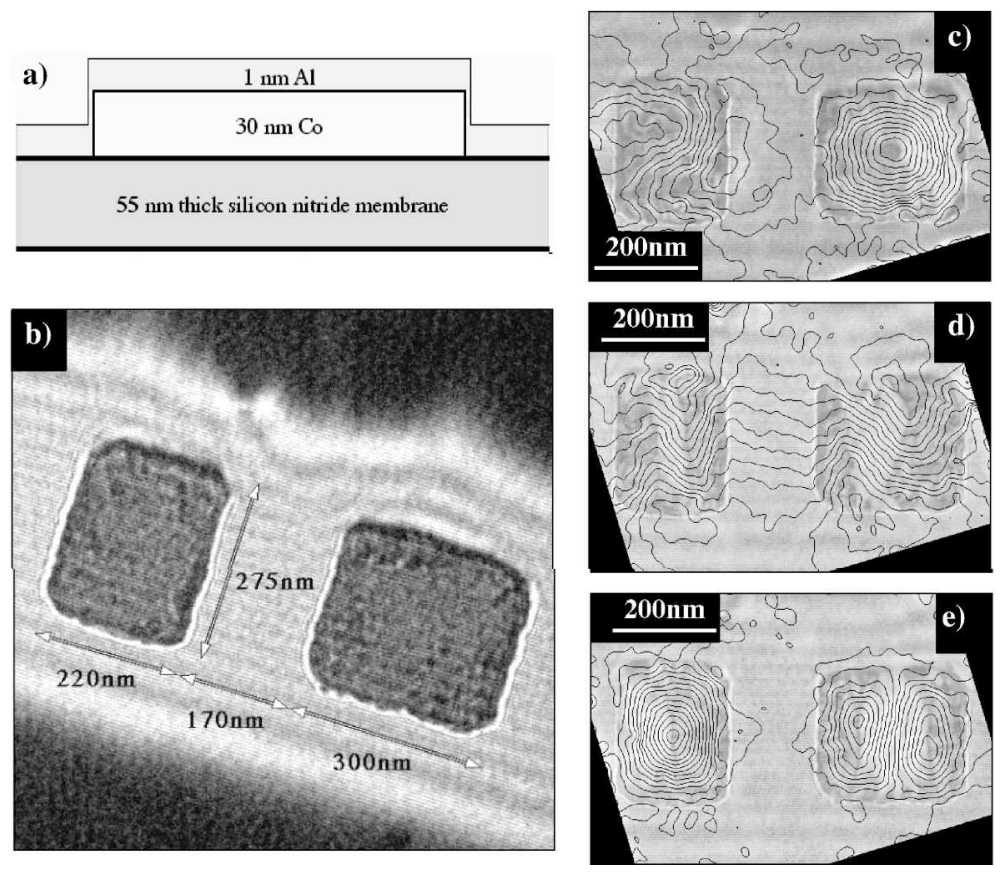

Fig. 9. a) Nominal cross-sectional geometry of $30-\mathrm{nm}$ -thick Co elements lithographically patterned onto a 55-nmthick electron-transparent silicon nitride membrane. b) Representative off-axis electron hologram of two patterned rectangular Co elements). c) - e) Magnetic contributions to phases of holograms showing three different remanent states of 30-nm-thick Co rectangles (with sample in small residual out-of-plane field of $13 \mathrm{mT}$ ). The contour spacing is $0.21 \pi$ radians.

magnetization of the constituent crystals. This parameter would be more difficult to measure for an isolated crystal without a model for the magnetic fringing field that surrounds it (de Graef et al., 1999). For the MS-1 cell examined above, the measured magnetic moment is consistent with that predicted for a chain of 2245 -nm diameter spheres if the saturation magnetization of bulk magnetite of $0.603 \mathrm{~T}$ $\left(480 \mathrm{emu} / \mathrm{cm}^{3}\right)$ is assumed and the stray fields that would be associated with isolated crystals are ignored. (These are negligible for a long chain). This result confirms that to within experimental error the remanent magnetization of the MS- 1 chain (the average magnetization parallel to the chain axis in zero applied field) is equal to the saturation magnetization of magnetite. This conclusion may not apply to crystals that have different compositions and easy axes that are not parallel to the chain axis. Similar measurements may also be used to measure values of magnetization that are still not known precisely, such as that of greigite $\left(\mathrm{Fe}_{3} \mathrm{~S}_{4}\right)$.

\section{Discussion and conclusions}

The well-defined shape and size of the magnetosomes synthesized by magnetotactic bacteria can be understood through a comparison of the present re- sults with the magnetic microstructure of slightly larger lithographically patterned elements. Fig. 9a shows the cross-sectional geometry of polycrystalline Co elements that have been deposited directly onto an electron-transparent silicon nitride membrane using electron beam lithography. A thin layer of $\mathrm{Al}$ is also deposited to prevent charging of the sample in the TEM. (This comparison is intended for illustrative purposes only; the magnetic properties of crystals of single-domain magnetite are clearly different from those of larger elements of polycrystalline Co). An off-axis electron hologram of two rectangular Co elements that are below $300 \mathrm{~nm}$ in size is shown in Fig. 9b, and measured magnetic contributions to the electron holographic phase are shown for three different remanent states in Fig. 9c-e. In contrast to the magnetic microstructure of biogenic magnetite crystals, several different domain structures can form within these elements, which are larger than the magnetic domain wall width in Co. As a result, the magnetic domain structure within the elements can rarely be reproduced between successive magnetization reversal cycles (Dunin-Borkowski et al., 2000). Furthermore, for a chain of such elements the presence of a complicated magnetic microstructure would result in a value for the remanent magnetization that is considerably smaller than the saturation magnetization. 
In this paper, we have shown that electron holography can be used to quantify the magnetic microstructure of a magnetosome chain in a magnetotactic bacterium, as well as the magnetic fringing field outside the cell, to a spatial resolution of better than $10 \mathrm{~nm}$. The magnetic contribution to the measured holographic phase can be separated from the compositional (mean inner potential) contribution to the contrast. Magnetite crystals in two strains of bacteria, denoted MV-1 and MS-1, were all found to be single magnetic domains. The magnetic moments and the coercive fields of individual cells were measured, and crystals that were smaller than the superparamagnetic limit were found to be magnetized as a result of interactions with larger crystals in the chains. The effect of shape anisotropy and magnetocrystalline anisotropy on the magnetic microstructure, as well as the reproducibility of the measured domain structure, were assessed. It will be of great interest to apply the technique to understand magnetite-based magnetic sensing mechanisms in higher organisms (Kirschvink \& Gould, 1981; Diebel et al., 2000; Winklhofer et al., 2001).

Acknowledgments: The authors are grateful to M.R. Scheinfein for discussions and grants from the NSF and NASA for support. Electron microscopy was carried out at the Center for High Resolution Electron Microscopy at Arizona State University. R.E.D. thanks the Royal Society for financial support.

\section{References}

Bazylinski, D.A., Frankel, R.B., Jannasch, H.W. (1988): Anaerobic magnetite production by a marine, magnetotactic bacterium. Nature, 334, 518-519.

Bazylinski,D.A., Garratt-Reed, A.J., Frankel, R.B. (1994): Electron microscopic studies of magnetosomes in magnetotactic bacteria. Microsc. Res. Tech., 27, 389-401.

Bazylinski, D.A. \& Moskowitz, B.M. (1997): Microbial biomineralization of magnetic minerals: microbiology, magnetism and environmental significance. Reviews in Mineralogy, 35, 181-223.

Blakemore, R.P. (1975): Magnetotactic bacteria. Science, 190, 377-379.

Chapman, J.N., Batson, P.E., Waddell, E.M., Ferrier, R.P. (1978): Direct determination of magnetic domain-wall profiles by differential phase-contrast microscopy. $U l$ tramicroscopy, 3, 203-214.

Daykin, A.C. \& Petford-Long, A.K. (1995): Quantitative mapping of the magnetic induction distribution using Foucault images formed in the transmission electron microscope. Ultramicroscopy, 58, 365-380.

de Graef, M., Nuhfer, T., McCartney, M.R. (1999): Phase contrast of spherical magnetic particles. J. Microsc., 194, 84-94. de Ruijter, W.J. \& Weiss, J.K. (1993): Detection limits for quantitative off-axis electron holography. Ultramicroscopy, 50, 269-283.

Diebel, C.E., Proksch, R., Green, C.R., Neilson, P., Walker, M.M. (2000): Magnetite defines a vertebrate magnetoreceptor. Nature, 406, 299-302.

Dunin-Borkowski, R.E., McCartney, M.R., Frankel, R.B., Bazylinski, D.A., Pósfai, M., Buseck, P.R. (1998b): Magnetic microstructure of magnetotactic bacteria by electron holography. Science, 282, 1868-1870.

Dunin-Borkowski, R.E., McCartney, M.R., Kardynal, B., Parkin, S.S.P., Scheinfein, M.R., Smith, D.J. (2000): Off-axis electron holography of patterned magnetic nanostructures. J. Microsc., 20, 187-204.

Dunin-Borkowski, R.E., McCartney, M.R., Kardynal, B., Smith, D.J. (1998a): Magnetic interactions within patterned cobalt nanostructures using off-axis electron holography. J.Appl. Phys., 84, 374-378.

Dunin-Borkowski, R.E., McCartney, M.R., Smith, D.J. (1998c): Off-axis electron holography of magnetic materials. Recent Res. Devel. in Applied Phys., 1, 119-131.

Dunin-Borkowski, R.E., McCartney, M.R., Smith, D.J., Parkin, S.S.P. (1998d): Towards quantitative electron holography of magnetic thin films using in situ magnetization reversal. Ultramicroscopy, 74, 61-73.

Dunlop, D.J. \& Özdemir, Ö. (1997): Rock magnetism: fundamentals and frontiers. Cambridge University Press, Cambridge.

Fabian, K.A., Kirchner, A., Williams,W. Heider,F, Leibl, T. Hubert, A. (1996): Three-dimensional micromagnetic calculations for magnetite using FFT. Geophys. J. Int., 124, 89-104.

Farina, M., Kachar, B., Lins, U., Broderick, R., Lins de Barros, H. (1994): The observation of large magnetite crystals from magnetotactic bacteria by electron and atomic force microscopy. J.Microsc., 173, 1-8.

Frankel, R.B. (1984): Magnetic guidance of organisms. Ann. Rev. Biophys. Bioeng., 13, 85-103.

Frankel,R.B., Bazylinski, D.A., Johnson,M.S., Taylor,B.L. (1997): Magneto-aerotaxis in marine, coccoid bacteria. Biophys.J., 73, 994-1000.

Jacobs, I.S. \& Bean, C.P. (1955): An approach to elongated fine-particle magnets. Phys. Rev., 100, 1060-1067.

Kirschvink, J.L. \& Gould, J.L. (1981): Biogenic magnetite as a basis for magnetic field sensitivity in animals. Biosystems, 13, 181-201.

Kuo,P.C. (1988): Chain-of-spheres calculation on the coercivity of elongated fine particles with both magnetocrystalline and shape anisotropy. J. Appl. Phys., 64, 5071-5083.

Lichte, H. (1991): Electron image plane off-axis holography of atomic structures. Adv. Opt. El.Micr. 12, 25-91.

Mann, S. (1993): Molecular tectonics in biomineralization and biomimetic materials chemistry. Nature, 365, 499-505.

Mann, S., Frankel, R.B., Blakemore, R.P. (1984): Structure, morphology and crystal growth of bacterial magnetite. Nature, 310, 405-407.

Mann, S., Sparks, N.H., Walker, M.M., Kirschvink, J.L. 
(1988): Ultrastructure, morphology and organization of biogenic magnetite from sockeye salmon, Oncorhynchus nerka: Implications for magnetoreception. J. Exp. Biol., 140, 35-49.

McKay, D.S., Gibson, E.K. Jr., Thomas-Keprta, K.L., Vali, H., Romanek, C.S., Clemett, S.J., Chillier, X.D.F., Maechling, C.R., Zare, R.N. (1996): Search for past life on Mars: Possible relic biogenic activity in Martian meteorite ALH84001. Science, 273, 924-930.

Meldrum, F.C., Mann, S., Heywood, B.R., Frankel, R.B., Bazylinski, D.A. (1993): Electron microscope study of magnetosomes in a cultured coccoid magnetotactic bacterium. Proc. Roy. Soc. Lond. B, 251, 231-236.

Moskowitz, B.M. (1995): Biomineralization of magnetic materials. Rev. Geophys. Suppl., 33, 123-128.

Moskowitz, B.M., Frankel, R.B., Bazylinski, D.A, Jannasch, H.W., Lovley, D.R. (1989): A comparison of magnetite particles produced anaerobically by magnetotactic and dissimilatory iron-reducing bacteria. Geophys. Res. Lett., 16, 665-668.

Penninga, I., de Waard, H., Moskowitz, B.M., Frankel, R.B. (1995): Remanence measurements on individual magnetotactic bacteria using pulsed magnetic fields. J.Magn.Magn.Mat., 149, 279-286.

Pósfai, M., Buseck, P.R., Bazylinski, D.A, Frankel, R.B. (1998): Reaction sequence of iron sulfide minerals in bacteria and their use as biomarkers. Science, 280, 880-883.

Proksch, R.B., Schaeffer, T.E, Moskowitz, B.M., Dahlberg, E.D., Bazylinski, D.A., Frankel, R.B. (1995): Magnetic force microscopy of the submicron magnetic assembly in a magnetotactic bacterium. Appl. Phys. Lett., 66, 2582-2584.
Reimer, L. (1991): Transmission electron microscopy. Springer, Berlin.

Saxton, W.O., Pitt, T.J., Horner, M. (1979): Digital image processing: the Semper system. Ultramicroscopy, $\mathbf{4}$, 343-354.

Smith, D.J., Dunin-Borkowski, R.E., McCartney, M.R., Kardynal, B., Scheinfein, M.R. (2000): Interlayer coupling within individual submicron magnetic elements. J. Appl. Phys., 87, 7400-7404.

Smith, D.J. \& McCartney, M.R. (1999): Practical electron holography. In Introduction to electron holography, eds. E. Völkl, L.F. Allard, D.C. Joy (Kluwer, New York), Chapter 4.

Thomas-Keprta, K.L., Bazylinski, D.A., Kirschvink, J.L., Clemett, S.J., McKay, D.S., Wentworth, S.J., Vali, H., Gibson,E.K.Jr., Romanek, C.S. (2000): Elongated prismatic magnetite crystals in ALH84001 carbonate globules: potential Martian magnetofossils. Geochim. Cosmochim. Acta, 64, 4049-4081.

Tonomura, A. (1992a): Electron-holographic interference microscopy. Adv. Phys., 41, 59-103.

- (1992b): Electron holography of magnetic materials and observation of flux-line dynamics. Ultramicroscopy, 47, 419-424.

Winklhofer, M., Holtkamp-Rötzler, E., Hanzlik, M., Fleissner, G., Petersen, N. (2001): Clusters of superparamagnetic magnetite particles in the upper-beak skin of homing pigeons: evidence for a magnetoreceptor. Eur. $J$. Mineral., 13, 659-669. 